Conclusion We have unmasked and rediscovered several exposures throughout the life course that associate with LVDD in later life, including dietary factors and cognition in childhood and young adulthood. Exposures identified in this study merit multi-cohort validation and have the potential to inspire more holistic public health efforts to tackle the emerging epidemic of HFpEF.

Conflict of Interest None

\section{CLINICIAN EXPERIENCES OF 1 YEAR OF TELEMEDICINE HEART FAILURE CLINICS: THE VIDEO-HF STUDY}

${ }^{1}$ Arvind Singhal, ${ }^{2}$ Jillian Riley, ${ }^{2}$ Martin Cowie. ${ }^{1}$ Royal Brompton Hospital, London, UK; ${ }^{2}$ Imperial College, London

\subsection{6/heartjnl-2021-BCS.116}

Background Prior to Covid-19, telemedicine clinics in heart failure (HF) were rare, but social distancing measures and increased demands on health services resulted in a shift to 'remote by default' clinic appointments in many organisations across Europe. We evaluated clinician experiences of telemedicine to determine its potential use post-Covid-19.

Methods From 16th March 2020 all HF appointments at a specialist centre were telemedicine by default, with in-person appointments only in exceptional circumstances. HF clinicians were invited to participate in semi-structured interviews about their experiences of telemedicine consultations. Interviews were conducted using Microsoft Teams, recorded and transcribed verbatim. Each interview lasted approximately 30 minutes. Narrative data were explored by thematic analysis: the iterative coding and comparison of transcripts to identify themes. Analysis was performed until themes saturated.

Results Between 16th March 2020 and 15th March 2021, there were $2725 \mathrm{HF}$ clinic appointments, 98.9\% of which were by telemedicine. 8 clinicians were interviewed: $4 \mathrm{HF}$ consultants, $3 \mathrm{HF}$ specialist nurses and one training-grade doctor. Four key themes emerged (figure 1): Time management telemedicine consultations were perceived to be more efficient, owing to more focused assessments, less time between appointments and ability to multitask, but more administrative and preparation time was required. Clinicians felt less guilty keeping consultations brief, as patients had not travelled for their appointment.Information gathering - without physical

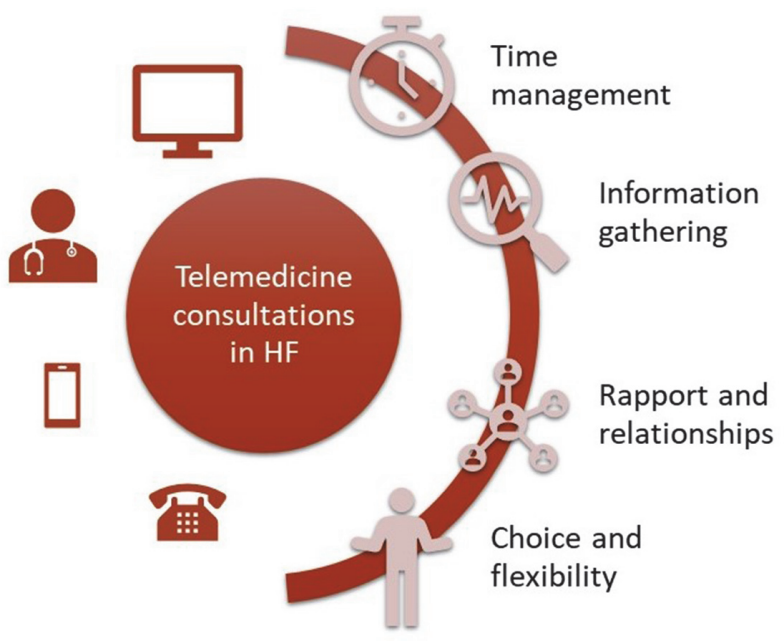

Abstract 119 Figure 1

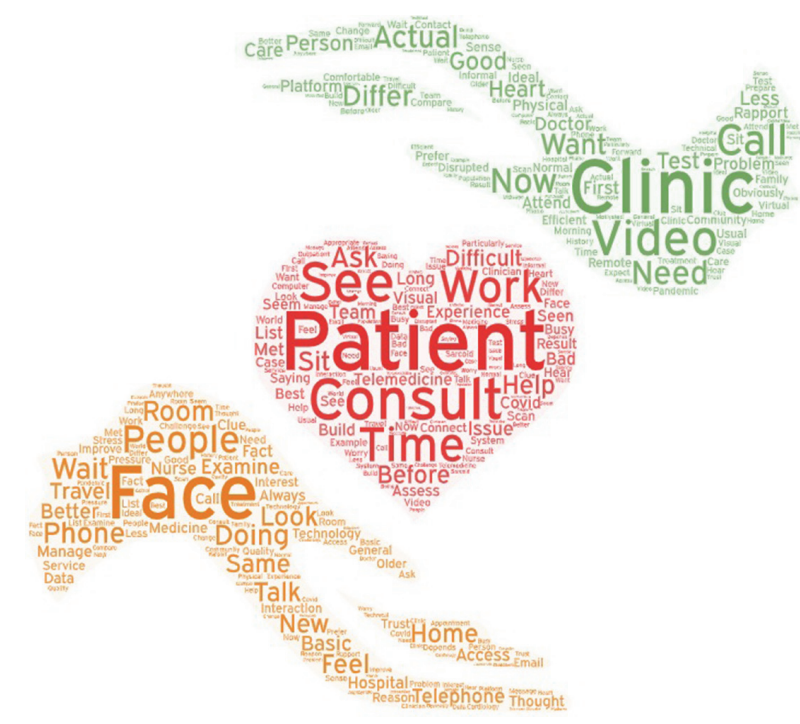

Abstract 119 Figure 2

examination clinicians relied more on objective data such as test results. Video was perceived as superior to telephone for assessing patients. Examination of oedema was possible by video, but more difficult and perceived to be less reliable.Rapport and relationships - telemedicine changed the patient-clinician interaction. Clinicians experienced difficulty establishing rapport with new patients by telephone; video was better than telephone, but clinicians felt that new patients were generally best assessed in-person to establish a 'connection' and relationship of trust.

Choice and flexibility - clinicians expressed a fear of 'topdown' diktats on future delivery of care. This was exemplified by the quote '...the health service has got a great tradition of making up its mind as to what the patient thinks'. Clinicians felt telemedicine consultations would continue to play a major role, as they were considered more convenient for patients, but patient choice was essential. Figure 2 shows a word cloud generated from interview transcripts.

Conclusions Telemedicine HF consultations were acceptable for clinicians, but changed workflows, consultation dynamics, and how clinicians developed rapport and trust. Understanding these changes is essential for future delivery of care. We will now seek to understand the views of patients and their families.

Conflict of Interest Dr Singhal's salary is funded by a fellowship from Abbott

\section{HOSPITAL ADMISSIONS IN THE LAST YEAR OF LIFE IN PATIENTS WITH HEART FAILURE}

${ }^{1}$ Alexandra Abel, ${ }^{1}$ Nathan Samuel, ${ }^{1}$ Joe Cuthbert, ${ }^{1}$ Syed Kazmi, ${ }^{2} J o h n$ Cleland, ${ }^{3}$ Andrew Clark. ${ }^{1}$ Hull University Teaching Hospitals NHS Trust, UK; ${ }^{2}$ University of Glasgow, UK; ${ }^{3}$ Hull York Medical School

\subsection{6/heartinl-2021-BCS.117}

Introduction In the last year of life, patients with heart failure (HF) may experience increasing symptoms and hospitalisation, but there are few data from UK populations. Whether there are differences between HF phenotypes in the pattern of admissions is not known. We explored the frequency, causes, 


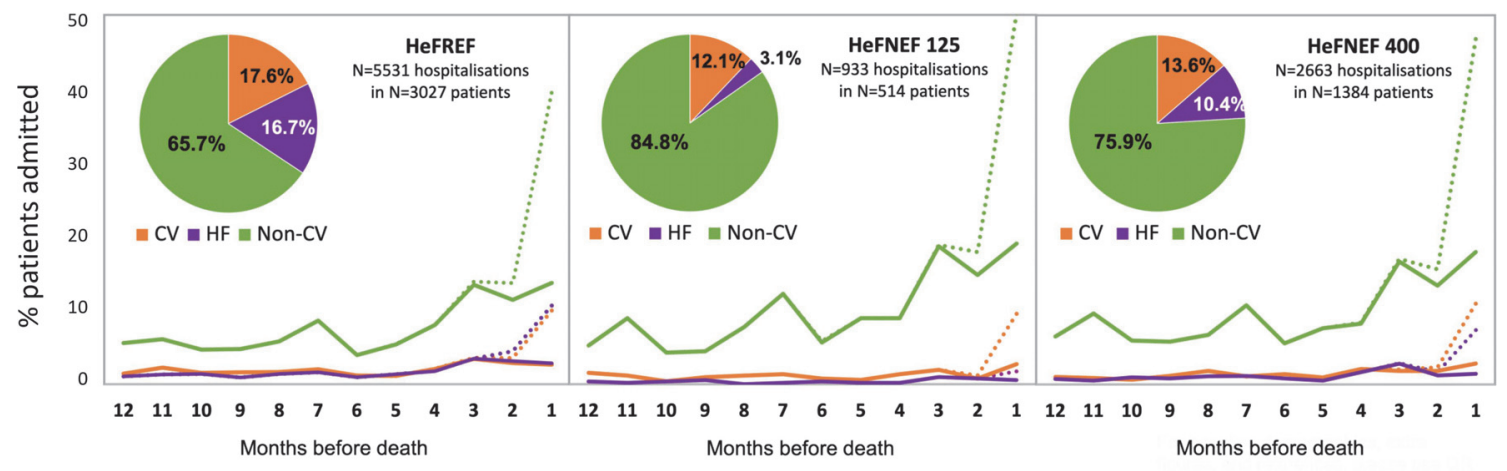

Bold lines: hospitalisations that resulted in discharge.

Dotted lines: hospitalisations where the patient died during admission.

Inset: Pie charts show the causes of admissions in the last year of life for each phenotype.

Abstract 120 Figure 1 Percentage of patients admitted each month prior to death

and pattern of hospitalisation for patients with $\mathrm{HF}$ in the 12 months preceding death.

Methods This is a retrospective analysis of patients recruited prospectively over two decades at a community HF clinic in Kingston upon Hull, UK. Patients with HF who died before August 2020 were included. We divided the cohort into three phenotypes: i) heart failure with reduced ejection fraction (HeFREF), ii) heart failure with normal ejection fraction (HeFNEF) with $\mathrm{N}$-terminal pro B-type natriuretic peptide (NTproBNP) 125-399 ng/L, and iii) HeFNEF with NTproBNP $\geq 400 \mathrm{ng} / \mathrm{L}$. The primary outcome was hospital admissions in the last year of life, classified as: HF, other cardiovascular (CV), or non-cardiovascular (non-CV). The primary cause of hospital admission using ICD-10 criteria was used.

Results Among 4925 patients, the median (Q1-Q3) age at death was 81 (75-87). $38 \%$ of patients were female. There were 9127 hospital admissions. The median (Q1-Q3) number of hospitalisations per patient was 2 (1-3) and days spent in hospital in the last year of life was 12 (2-25). 83\% of patients had at least one hospitalisation; $20 \%$ had at least one HF hospitalisation; 24\% had at least one CV hospitalisation; $70 \%$ had at least one non-CV hospitalisation. 54\% of deaths were as an in-patient. HF admissions were a minority in all phenotypes, but most common in HeFREF. In each group, at least two thirds of admissions were non-CV. $6 \%$ of the admissions in 2020 (prior to August) were for COVID-19. Hospitalisations increased in the three months prior to death (figure 1).

Conclusion In the last year of life in patients with HF, most hospitalisations are for non-CV causes regardless of $\mathrm{HF}$ phenotype.

Conflict of Interest None

\section{EFFECT OF UK COVID-19 PUBLIC HEALTH MEASURES ON ACTIVITY AND QUALITY OF LIFE IN PATIENTS WITH PULMONARY ARTERIAL HYPERTENSION}

${ }^{1}$ Jennifer Middleton, ${ }^{1}$ Hamza Zafar, ${ }^{2}$ Ashwin Reddy, ${ }^{3}$ Claire Martin, ${ }^{1}$ Roger Thompson, ${ }^{1}$ Andrew Swift, ${ }^{2}$ Mark Toshner, ${ }^{1}$ David.G Kiely, ${ }^{1}$ Alexander Rothman. ${ }^{1}$ University of Sheffield, Sheffield, UK; ${ }^{2}$ University of Cambridge; ${ }^{3}$ Royal Papworth NHS Foundation Trust

10.1136/heartjnl-2021-BCS.118

Background Limitation of activity and restriction of movement have been widely, and effectively, enforced to reduce COVID-
19 transmission. Physical activity is however a critical measure in the prevention of cardiovascular disease. Pulmonary arterial hypertension $(\mathrm{PAH})$ is a devastating, disease driven by small vessel vascular remodeling, leading to right heart failure. Exercise capacity relates to clinical outcomes and exercise training improves key indicators of cardiopulmonary function. Here, we describe the temporal effects of UK government restriction measures on daily activity, heart rate and quality-of-life (QoL) in patients with PAH.

Methods From November 2019 to March 2020 patients were enrolled into the arrhythmia sub-study of The UK National Cohort Study of Idiopathic and Heritable PAH (REC:13/EE/ 0203) and implanted with insertable cardiac monitors. Daily heart rate, heart rate variability and activity were transmitted remotely. Standard questionnaires were administered remotely to assess QoL (EmPHasis-10), anxiety (GAD-7) and depression (PHQ-9).

Results Median age of the 26 patients implanted with insertable cardiac monitors was 49 years, 23(88\%) were female and $5(19 \%)$ had heritable PAH with mutations in BMPR2. At enrolment $10(38.5 \%)$ patients were low risk $(<5 \%$ 1-year mortality), $10(38.5 \%)$ were intermediate risk $(5-10 \%)$ and 6 (23\%) were high risk (>10\%). The mean duration from insertion to census date was 21.1weeks \pm 5.7 . No complications were reported. Completeness of remote monitoring data was 100\%. Following lockdown, mean activity was reduced (3.16vrs 2.68hours, -0.48 hours, 95\%CI -0.27-0.69, 16\%, $\mathrm{p}<0.0001)$. During the period April 14th to 23rd QoL was reduced $(26(18-38)$ vrs $32(17-47), \mathrm{p}<0.01)$ and anxiety $(1(0-$ 9)vrs $10(5-18), \mathrm{p}<0.001)$ and depression scores increased (3 $(1-16)$ vrs 11 (3-17), p<0.001) compared to pre-lockdown levels. The observed increase in depression scores persisted to the census date $(3(1-16)$ vrs $11(8-17), \mathrm{p}<0.01)$. No change in day or night heart rate, or heart rate variability, was observed and no patients developed COVID-19.

Conclusion In this cohort of patients with idiopathic and heritable PAH, UK protective health measures were effective in preventing COVID-19 in patients thought to be vulnerable. However, these protective measures resulted in reduced daily activity and QoL and were associated increased anxiety and depression indicators. Patients may decondition through periods of reduced activity. This may have implications for riskassessment and endpoint adjudication in clinical studies, both of which use measures of exercise capacity.

Conflict of Interest none 